\title{
Transient therapy with quadruple NRTI provides immune stability in patients with multidrug resistant HIV-I and no options for suppressive regimens
}

\author{
A Bonjoch*1, JM Llibre ${ }^{1}$, E Negredo ${ }^{1}$, J Puig1, N Pérez-Álvarez1, MJ Buzon², \\ J Martinez-Picado ${ }^{2}$ and B Clotet ${ }^{3}$
}

\begin{abstract}
Address: ${ }^{1}$ Lluita SIDA Found Germans Trias i Pujol University Hospital, Badalona, Spain, ${ }^{2}$ IrsiCaixa Foundation, Barcelona, Spani and ${ }^{3}$ Lluita Contra la Sida and IrsiCaixa Foundation, Barcelona, Spain

* Corresponding author
\end{abstract}

from Ninth International Congress on Drug Therapy in HIV Infection

Glasgow, UK. 9-13 November 2008

Published: 10 November 2008

Journal of the International AIDS Society 2008, I I (SuppI I):P50 doi:I0.I I86/I758-2652-I I-SI-P50

This abstract is available from: http://www.jiasociety.org/content/II/SI/P50

(C) 2008 Bonjoch et al; licensee BioMed Central Ltd.

\section{Purpose of the study}

Preventing immunological deterioration is essential in highly-experienced HIV-1-infected patients who have no options for fully suppressive therapies. We explored a holding regimen aimed to preserve the immunologic status of patients with multidrug resistant virus while awaiting new active drugs.

\section{Methods}

Exploratory, randomized study in heavily pretreated patients on failing therapies. The objective was to determine if a holding regimen integrated by co-formulated zidovudine/lamivudine/abacavir and tenofovir was able to maintain immunological status. Virologic outcomes, genotype evolution and clinical safety were also evaluated. Control arm received a genotype-guided salvage regimen.

\section{Summary of results}

Twenty-three patients (pts) were recruited; 13 were assigned to the holding-arm and 10 to the control-arm. $76 \%$ and $80 \%$, respectively, had $\geq 3$ TAMs at baseline (BL). Genotype sensitive score was $0.5(0.5 ; 0.75)$ and $0.5(0.5$; 1), respectively. Median (IQR) BL CD4 count was 366 $(293 ; 448)$ in holding- and $420(189 ; 456)$ cells $/ \mu \mathrm{L}$ in control-arm ( $\mathrm{p}=0.9)$, and median BL viral load $(\mathrm{VL})$ was $3.5(3 ; 4)$ and $4.12 \log 10(3.3 ; 4.5)$, respectively. After 48 weeks, $62 \%$ in the holding- and $100 \%$ of the control-arm maintained their CD4 count $(\mathrm{p}=0.09)$. VL decreased a median of $0.6(0.2 ; 1.9)$ and $2 \log 10(0.5 ; 2.7)$ in the holding- and control-arm $(\mathrm{p}=0.1) .27 \%$ of the pts in the holding- and 50\% in control-arm achieved undetectable VL. Number of TAMs and mutations in the protease gene were maintained in both groups. No clinical progression was observed. Adverse events were detected in $16 \%$ in holding- and $90 \%$ in control-arm, respectively ( $\mathrm{p}=$ 0.001 ). When a fully suppressive therapy could be initiated, $69 \%$ of the pts from holding-arm (nine subjects) and $60 \%$ (six pts) from the control-arm achieved $\mathrm{VL}<50$ copies at 48 weeks of follow-up after the study ending.

\section{Conclusion}

Our results showed better virological and immunological outcomes with standard salvage therapy than a holding therapy with TZV+TDF. However, this approach provided a stable immunologic status, better tolerability and it was not associated with clinical progression for 48 weeks of follow-up. This strategy did not jeopardize a posterior complete viral suppression when a fully active regimen could be initiated. This transient approach could be useful in pts with multidrug resistant HIV-1, toxicities or other condition that restrict active drugs while awaiting a fully suppressive regimen. 